\title{
2 DOF Low Cost Platform for Driving Simulator: Modeling and Control
}

\author{
Hichem Arioui, Salim Hima and Lamri Nehaoua
}

\begin{abstract}
In order to be an effective tool for driver evaluation and education, driving simulators need to be better designed to reduce simulator sickness. In this paper, we expose platform design, description and the modeling aspects of a 2 DOF low cost motion platform allowing the restitution of the longitudinal and yaw movements. To enhance the drive immersion in the virtual world, a haptic feedback steering wheel will be implemented.

The whole system is considered as a two coupled systems and linked mechanically. The first system consists in motorized rail for the longitudinal movement while the second system consists in motorized yaw allowing either curve-taking movement.

The platform mechanics is proposed as presented in the next sections to study the driving simulator sickness on the driver and especially the yaw component. Experimental studies were made to devise a characterization of the platform capabilities and frequency responses. Experimentations were carried out for classical drive operation. First conclusion and future works are established.
\end{abstract}

Index Terms-Driving Simulator design, Dynamics and Modeling.

\section{INTRODUCTION}

The use of driving simulators is increasingly widespread and adopted by various public and private institutions. A driving simulator is virtual reality tool allows users to drive in safe way and test several scenarios on same system. These motion cueing platforms were firstly used for aircraft simulators and were democratized for cars and recently for motorcycle [1][2][3]. Driving simulators became very accessible by technological headway. Indeed, the calculators become more powerful and less expensive. Thus, several simulators of various architectures were built with an aim of either human factor study [4][5][6][7], or vehicle dynamic model validation, or test of new car prototypes and functionalities [8][9][10].

However, if the cost of aircraft and passenger safety allow investment in high-cost simulators, nothing justifies it or even provides a tool for training and psychophysical studies in the car [11][12] and motorcycle cases [13]. In addition, the complexity of a simulator does not reflect its fidelity to feed back all the movements. For this reason, it was interesting to offer targeted solutions based low-cost mobile platforms aimed primarily at training schools, hospitals and other users.

Furthermore, driving simulator system allows a driver to interact in a safe manner, with a synthetic urban or highway via a motion cueing platform by feeding back the essential

This work was supported by French National Agency of Research (ANR) in the Framework of VIGISIM Project.

H. Arioui, S. Hima and L. Nehaoua are with IBISC-CNRS Fre3190 at University of Evry Val d'Essonne, 40, rue du Pelvoux, CE1455 Courcouronnes, Evry cedex, France. Email : hichem.arioui@ibisc.fr inertial components (acceleration and rotation) to immerse driver partially or completely. Indeed, this clearly means that as the complexity of such experiments is lies in the fact that the simulation is composed of interconnected subsystems of different nature (biological, mechatronics, control laws, computer, etc.) and should be studied in its entirety. In this work, we are interested by the design, mechactronics and identification parts of the platform.

More generally, on motion cueing platform, a large range of real-driving experienced accelerations cannot be reproduced. A compromise is to be found between the quality of various inertial indices' restitution and maintaining the platform within its reachable workspace. Therefore, many control strategies were developed [14][15][16][17], [18]. The Motion Cueing Algorithms (MCA) were firstly used for flight simulators motion cueing. Their porting to vehicle simulators is possible, but the vehicle dynamics is of much higher frequencies (more abrupt and frequent acceleration variation) than what is observed on airplanes. Besides, driving a vehicle takes place within traffic and unforeseen events (fog, pedestrians...) conditions which could create more complex scenarios.

All the components cited before will be taken into account in order to facilitate the design built a low-cost motion platform equipped with two degrees of freedom.

In the rest of this paper we present the design, description and modeling aspects of the platform, followed by the experiments that were carried to characterize frequentially the motions. We finish this present work by the traditional conclusion and future works.

\section{Choices' Motivation of the Platform ARCHITECTURE}

The simulator structure and motions based choices are motivated by the necessary needs to have a sufficient perception while riding under financial constraint to make easy the duplication in favor of driving schools and other institutions. In this sense, the objective of the simulator project is not to reproduce the whole vehicle motions, but only the longitudinal and yaw one. These inertial effects are to be perceived by the human user for the expected applications which aims to study the effect of yaw component on simulator sickness.

Because of the importance of accelerating transition motion in vehicle dynamics, we also emphasize the longitudinal movement.

Moreover, we know that the multiplication of perception stimuli can strongly increase the riding simulation sensations [19]. Based on this observation, the reproduction of the dynamic tire-road contact system to assist the driver is 
implemented (actuator mounted on the steering wheel). The modeling part of this last point is not addressed in the present paper. Figure (1) presents the experimented architecture platform which will be described in the next section.

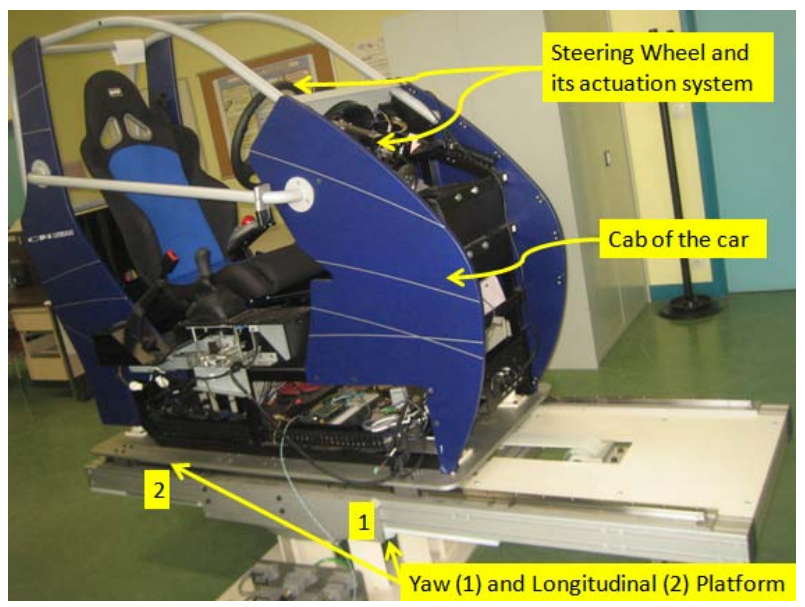

Fig. 1. The experimented 2 DOF simulator platform.

\section{PlATFORM DESCRIPTION}

\section{A. Simulator Architecture}

We present in this paper a mini driving simulator with an acceptable compromise between the quality of restitution, compactness and under cost constraints. The mechatronics components of the proposed solution are described below:

- The cabin consists of an instrumented mobile part moving along a guide-way mounted on the platform. It is the interface that lies between the driver and the simulation environment. The cabin is equipped with acceleration and braking pedals, steering wheel, gearbox lever and other classical car implements which are having appropriate sensors that allow the acquisition of the driver desired input commands (figure 1). These inputs feed the vehicle dynamic model to update its several states. The cabin disposes also of different visual indicators rendering the engine rpm, the vehicle speed, etc.

- The acquisition system is composed of an industrial micro controller, and has both analog and digital input/output. This allows the control of the actuators in the desired position, speed or torque; this card appeared to be well adapted for the interfacing of the simulator's cabin. A bidirectional information exchange protocol is settled between this card and the PCs dedicated to vehicle-traffic model. This can be performed either through a parallel or a CAN ports.

- The vehicle model concerns the computation of the dynamics and the kinematics according to the driver actions such as acceleration and brake pedals' positions, clutch... that are transmitted through the acquisition module and the road characteristics. It is a simple model dedicated to our simulator driving application. In this model, the vehicle is considered as one body with 5DOF (longitudinal, lateral, roll, pitch and yaw). Its complexity relates more to the motorization part than the chassis dynamic. The engine part is modeled by a mechanical and behavioral approaches [20] based on the vehicle general characteristics (engine torque curves, clutch pedal position, accelerating proportioning, etc). After updating the vehicle's state, resulting information on the engine are sent to the cabin's dashboard and to the traffic model server.

The traffic model, visual and audio systems are not yet operational on this platform.

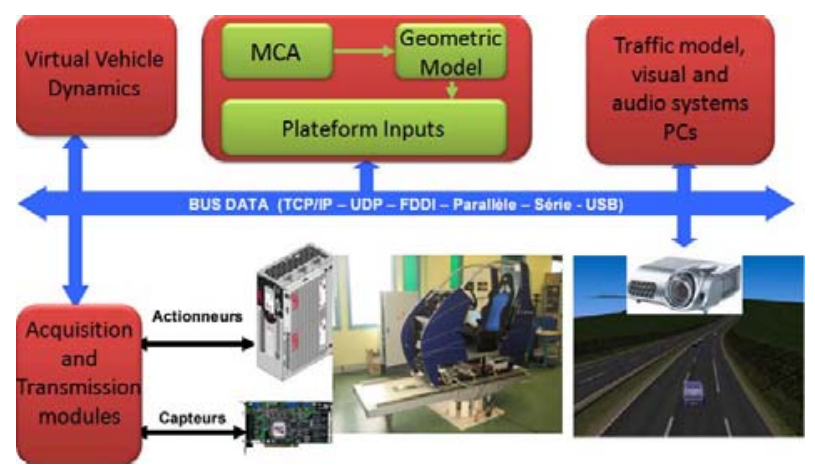

Fig. 2. Simulation Synoptic Architecture.

The platform is embedded with power, sensors and control modules to have information feedback on the control system states. Each actuator (for the yaw and longitudinal one) has servomotor level to ensure angular position transducer, angular velocity and the output torque's estimation. Data resulting from these module are sent to the input/output interface board that is managed by a control PC transmitted via CAN technology.

\section{B. Mechanical Description}

The platform is composed of two metallic parts linked mechanically. The upper part is composed by the cabin car supported by chassis moving longitudinally on the lower part. This last one consists of horizontal structure on which is fixed the rotation drive system of the yaw motion (see figures 3 and 4). Overall upper system: cabin, driver and the sliding plate have an average weight of $380 \mathrm{~kg}$.

To control yaw and longitudinal motions of the platform, two actuators have been used. Through two sliders, assembled under the two edges of the cabin's base, the platform is able to move on a rail of $1.2 \mathrm{~m}$ length. To this end, a Brushless type motor SMB 80 (nominal and peak torques are respectively 3 and $9 \mathrm{~N} . \mathrm{m}$ ) with a reduction ratio of 45 are fixed at a mechanical stand related to the platform's rails. The motor rotation is transformed into cabin's longitudinal motion through a pulley-belt system. This platform achieves linear accelerations up to $\pm 0.408 \mathrm{~g}$ in steady mode. At peak current, acceleration and speed of $\pm 1.224 \mathrm{~g}$ and \pm 2.45 $\mathrm{m} / \mathrm{sec}$ respectively are reached. The SMB 80 actuator is driven with a brushless type servomotor. 


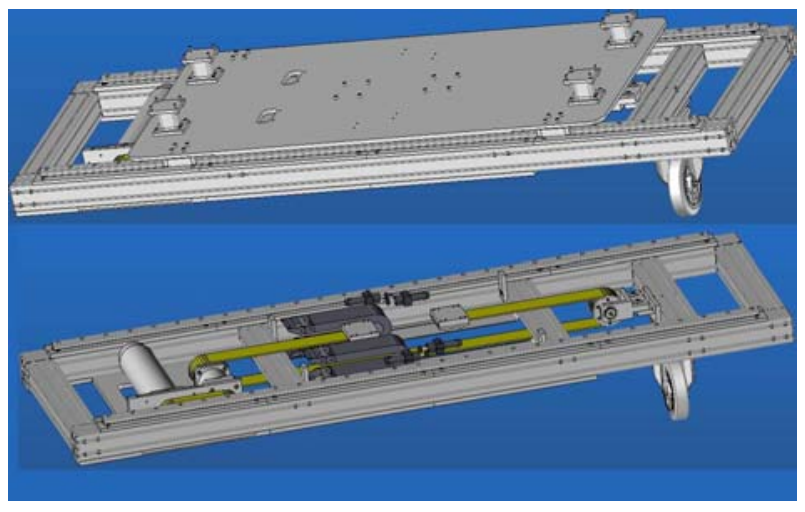

Fig. 3. Upper metallic frame of the longitudinal motion

On the yaw motion, it is directly controlled by placing a rotation system under the vertical structure and driven by a circular ball-screw drive actuated system (the same actuator, SMB 80 , used for the longitudinal motion) operated by a brushless servomotor and reduction red of 139.2 see figure 4. The motor rotation is transformed into ball-screw system through a right angle transformation. This system achieves angular accelerations up to $3.971 \% / s^{2}$ in steady mode. At peak current, acceleration and speed of $58.151 \% / s^{2}$ and $29.075 \%$ respectively are reached.

On the present driving simulator, the yaw rotation of the platform can be changed or adapted to the situation that we want simulate. Indeed, the upper part of the platform moves manually over the lower part. This option allows us to study better the impact of the rotation yaw (rotation speed and radius of curvature, etc.) on the perception quality of the restituted motion.

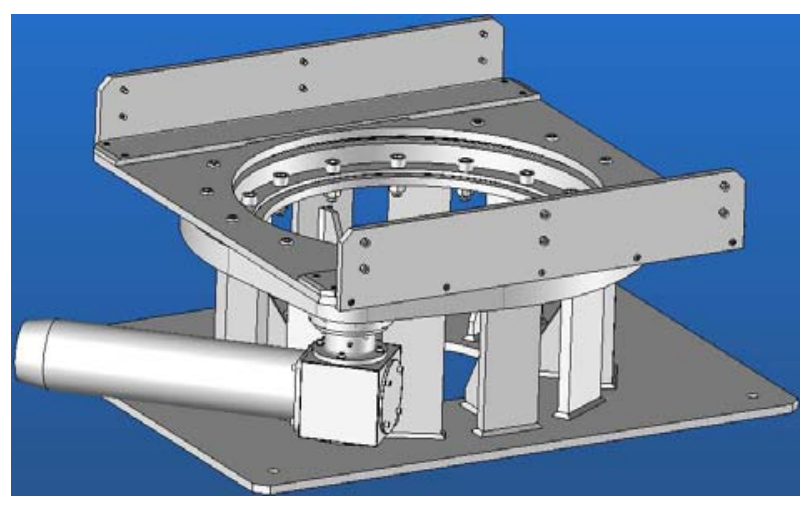

Fig. 4. The circular ball-screw drive actuated system

\section{Platform Modeling and Identification}

Mainly, control of robotic mechanisms is based on the knowledge of an accurate behavioral model that governs their motions. Indeed, the accuracy of the model depends essentially on the quantification of the phenomena that acts on it, and on the precision of its parameters. We devote this section to the derivation of the dynamic model of our platform in response to actuators torques.

\section{A. Platform Kinematic Modeling}

The effect of the front wheel dynamics on that of the whole system is neglected. Hence, by removing the wheel and replace it with a resistive torque, resulting from the friction forces of the wheel/ground interaction, and acting on the yaw motion, the system treated in this paper can be seen as a serial multi body system with three bodies linked by two degrees of freedom, RP manipulator. In this case, Three orthonormal frames are used to describe the motion of the platform, see figure 5. Body $B_{0}$ and body $B_{1}$ are linked with a revolute joint parametrized by the variable $q_{1}$. So, the transformation between frame $\mathcal{R}_{0}$ and $\mathcal{R}_{1}$ is given by the rotation matrix:

$$
R=\left(\begin{array}{ccc}
\cos \left(q_{1}\right) & -\sin \left(q_{1}\right) & 0 \\
\sin \left(q_{1}\right) & \cos \left(q_{1}\right) & 0 \\
0 & 0 & 1
\end{array}\right)
$$

Besides, Body $B_{2}$ performs a translation with respect to body $B_{1}$ parametrized by the variable $q_{2}$. Hence, the configuration of the platform can be easily described by the vector $\mathbf{q}=\left(q_{1}, q_{2}\right)$.

Let $\left(x_{G_{1}}, y_{G_{1}}, z_{G_{1}}\right)$ and $\left(x_{G_{2}}, y_{G_{2}}, z_{G_{2}}\right)$ denote, respectively, the positions of the center of mass for bodies $B_{1}$ and $B_{2}$ in their attached frames.

Angular velocities of bodies $B_{1}$ and $B_{2}$ are given by:

$$
\omega_{1}=\omega_{2}=\left(\begin{array}{c}
0 \\
0 \\
\dot{q}_{1}
\end{array}\right)
$$

Then linear velocities of $G_{1}$ and $G_{2}$ are given by:

$$
\begin{aligned}
\mathbf{V}_{G_{1}} & =\omega_{1} \times \mathbf{O}_{1} \mathbf{G}_{1} \\
\mathbf{V}_{G_{2}} & =\mathbf{V}_{O_{2}}+\omega_{2} \times \mathbf{O}_{2} \mathbf{G}_{2}
\end{aligned}
$$

By projecting these expression in their local frames we can find:

$$
\begin{gathered}
\mathbf{V}_{G_{1}}=\left(\begin{array}{c}
-\dot{q}_{1} y_{G_{1}} \\
\dot{q}_{1} x_{G_{1}} \\
0
\end{array}\right) \\
\mathbf{V}_{G_{2}}=\left(\begin{array}{c}
-\dot{q}_{1} y_{G_{2}}+\dot{q}_{2} \\
\left(x_{G_{1}}+q_{2}\right) \dot{q}_{1} \\
0
\end{array}\right)
\end{gathered}
$$




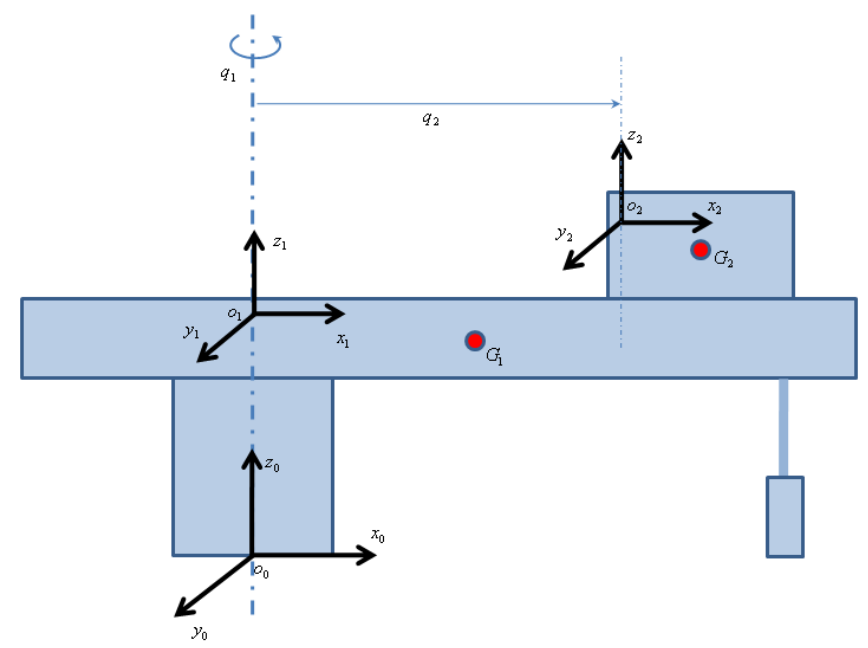

Fig. 5. Platform frames description

\section{B. Platform Dynamics}

Modeling mechanical mechanisms have attracted a great attention for a long time and have attained a great maturity. In fact, these development have led to a very efficient algorithms which are accurate and rapid in order to fulfilling requirements for robotic applications or computer animation for example and for a large degrees of freedom [21].

There exist several methods to derive the dynamics equations of mechanisms such as: Newton-Euler's formalism, Hamilton's formalism, Kane's formalism ... etc. In this paper, we have used the Lagrange's formalism for its simplicity. The equations of motion can than be obtained using Lagrange's equation for each generalized coordinates:

$$
\frac{d}{d t}\left(\frac{\partial T}{\partial \dot{q}_{i}}\right)-\frac{\partial T}{\partial q_{i}}=Q_{i}
$$

where $T=V-U$ is the Lagrangian function defined by the difference between the total kinetic energy of the system $V$ and the total potential energy of the system $U$. In the case of the platform presented in this paper, which evolves in $(X Y)$ plan, $T$ is reduced only to kinetic energy:

$$
T=V=\frac{1}{2} \sum_{i=1}^{2}\left(\mathbf{V}_{G_{i}}^{T} \mathbf{M}_{i} \mathbf{V}_{G_{i}}+\omega_{i}^{T} \mathbf{I}_{i} \omega_{i}\right)
$$

where $\mathbf{M}_{\mathbf{1}}=m_{1}$ eye $(3)$ and $\mathbf{M}_{\mathbf{2}}=m_{2}$ eye(3) are respectively, body $B_{1}$ and $B_{2}$ matrix of masses, $\mathbf{I}_{\mathbf{1}}=$ $\operatorname{diag}\left(I_{1_{x x}}, I_{1_{y y}}, I_{1_{z z}}\right)$ and $\mathbf{I}_{\mathbf{2}}=\operatorname{diag}\left(I_{2_{x x}}, I_{2_{y y}}, I_{2_{z z}}\right)$ are respectively, the moment of inertia tensors of bodies $B_{1}$ and $B_{2}$ expressed in their local frames.

By replacing equations (4) and (5) in equation (7), the previous expression becomes:

$$
\begin{aligned}
T= & \frac{1}{2}\left(m_{1}\left(x_{G_{1}}^{2}+y_{G_{1}}^{2}\right)+I_{1_{z z}}+m_{2} y_{G_{2}}^{2}+m_{2}\left(x_{G_{1}}+q_{2}\right)^{2}\right. \\
& \left.+I_{2 z z}\right) \dot{q}_{1}^{2}+\frac{1}{2} m_{2} \dot{q}_{2}^{2}-m_{2} y_{G_{2}} \dot{q}_{1} \dot{q}_{2}
\end{aligned}
$$

It is a straightforward to show that, by application of Lagrange's formalism, equation (6), the platform equations of motion can take the following from:

$$
\mathbf{M}(\mathbf{q}) \ddot{\mathbf{q}}+\mathbf{C}(\mathbf{q}, \dot{\mathbf{q}})=\mathbf{Q}
$$

where $\mathbf{M}(\mathbf{q})$ is the system inertia matrix given by:

$$
\mathbf{M}(\mathbf{q})=\left(\begin{array}{ll}
m_{11} & m_{12} \\
m_{21} & m_{22}
\end{array}\right)
$$

such as:

$$
\begin{aligned}
m_{11}= & m_{1}\left(x_{G_{1}}^{2}+y_{G_{1}}^{2}\right)+I_{1_{z z}} \\
& +m_{2} y_{G_{2}}^{2}+m_{2}\left(x_{G_{2}}+q_{2}\right)^{2}+I_{2_{z z}} \\
m_{12}= & m_{21}=-m_{2} y_{G_{2}} \\
m_{22}= & m_{2}
\end{aligned}
$$

and $\mathbf{C}(\mathbf{q}, \dot{\mathbf{q}})$ is a vector of centrifugal and Coriolis forces:

$$
\mathbf{C}(\mathbf{q}, \dot{\mathbf{q}})=\left(\begin{array}{c}
2 m_{2}\left(x_{G_{2}}+q_{2}\right) \dot{q}_{1} \dot{q}_{2} \\
-m_{2}\left(x_{G_{2}}+q_{2}\right) \dot{q}_{1}^{2}
\end{array}\right)
$$

$\mathrm{Q}$ is the external forces/torques vector acting on the platform including traction and friction forces/torques:

$$
\mathbf{Q}=\left(\begin{array}{c}
\tau_{1}-\tau_{f_{1}} \\
\tau_{2}-\tau_{f_{2}}
\end{array}\right)
$$

$\tau_{1}$ and $\tau_{2}$ are obtained by multiplying the effective torque delivered by motors, by the reduction ration of gears systems integrated into their corresponding joints:

$$
\begin{aligned}
& \tau_{1}=K_{1} \tau_{a_{1}} \\
& \tau_{2}=K_{2} \tau_{a_{2}}
\end{aligned}
$$

where : $K_{1}$ and $K_{2}$ are respectively about 139.2 and 45 . $\tau_{f_{1}}$ and $\tau_{f_{2}}$ are the friction torques. These torques are modeled as a combination of dry and viscous frictions:

$$
\tau_{f_{i}}=K_{s_{i}} \operatorname{sign}\left(\dot{q}_{i}\right)+K_{v_{i}} \dot{q}_{i} \quad i=1,2
$$

In next section, we will discuss the all parameters identification of the developed dynamic model and the used approach. The frequency characterization of the system dynamic is also done to make an idea on the system transitions capabilities.

\section{EXPERIMENTAL RESUlts}

In this section we present the results of tests made on the simulator for its frequency characterization. For the purpose of experiments, a PID controllers are used to control each of platform articulations $\left(q_{1}, q_{2}\right)^{T}$.

Firstly, we are looking for frequency characteristics of longitudinal and yaw motions. To do this, the system have being excited with a chirp signal (sine wave with increasing frequency) independently axel by axel. The resultant inputouput signals is used to identify the parameters the parameters of the linear system which match this motion, by using the least square method. Hence, the obtained system is used to identify the bandwidth of the corresponding motion.

Figure 6 and 7 represent the experimental measures of the longitudinal and yaw motions respectively when excited by the chirp signal. The best fit is obtained by second order and third order system for respectively the longitudinal and yaw 
motions. It is clear that proceeding by this way can give just an initial overview of the platform frequency capabilities. In the future works, we look to examine a combined motions, longitudinal and yaw in the same time, which can brings out the non-linear phenomena, and so, gives a more accurate values of the system's frequency capabilities.
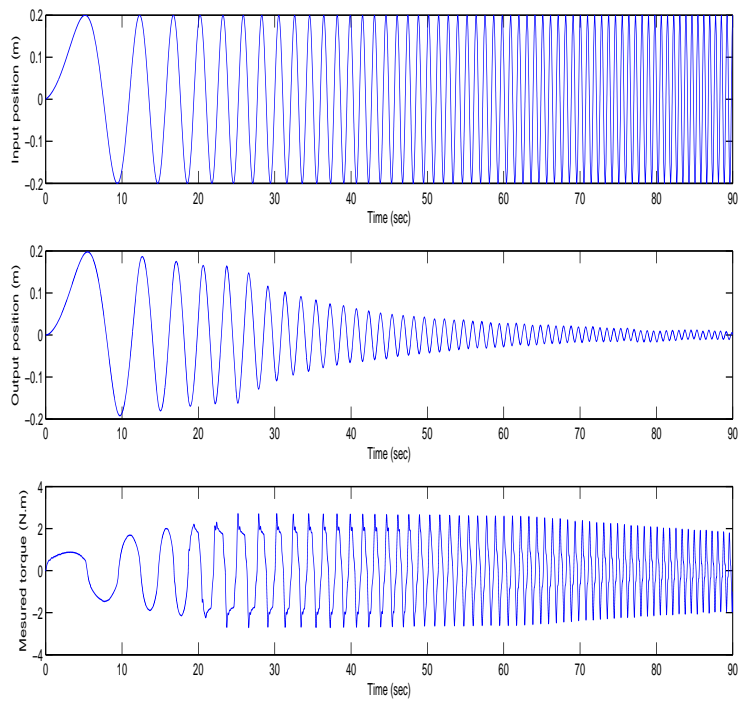

Fig. 6. Platform longitudinal motion response to chirp sine input
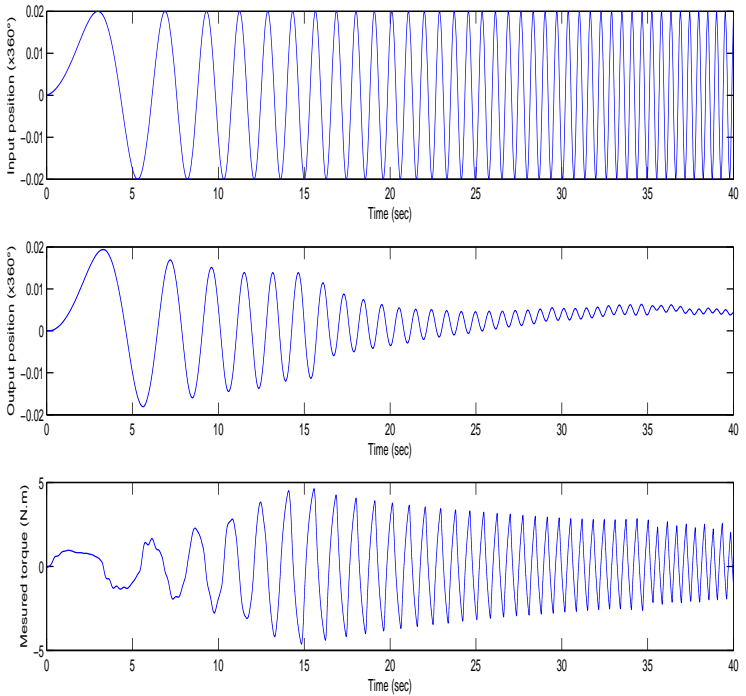

Fig. 7. Platform yaw motion response to chirp sine input

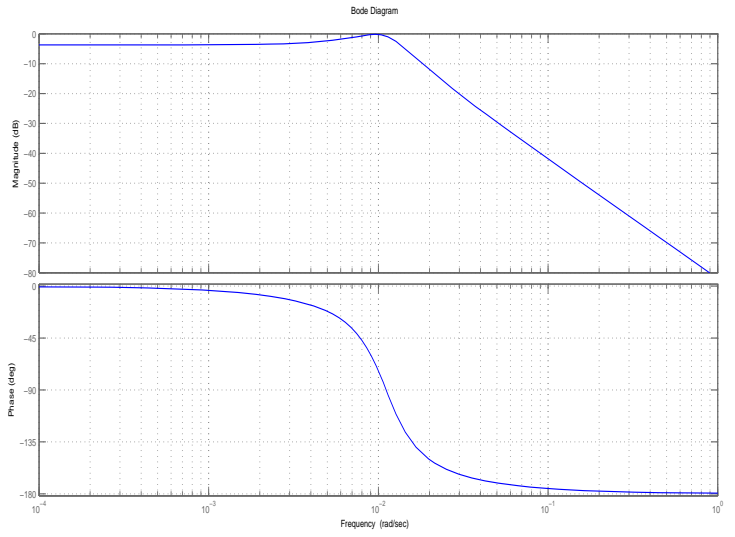

Fig. 8. Bode diagram of the longitudinal motion

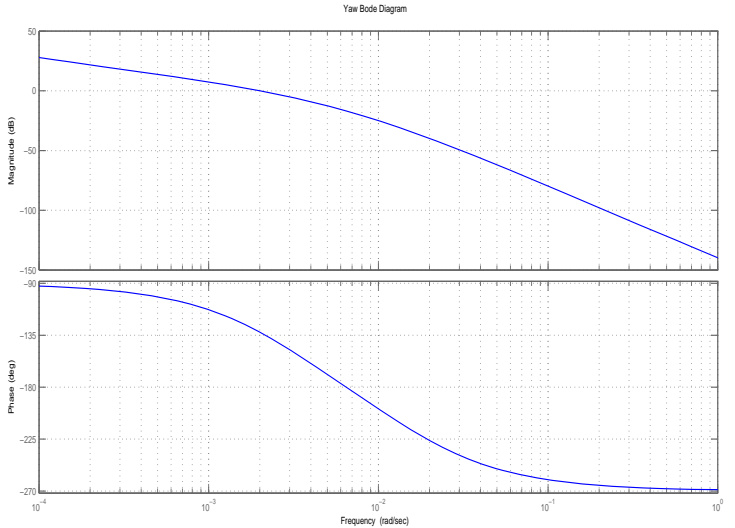

Fig. 9. Bode diagram of the yaw motion

Figure 8 and 9 present Bode diagrams of the longitudinal and yaw motion respectively. Form these figures, we can find that, the cut frequency of the longitudinal motion is about $\omega_{c l}=0.2(\mathrm{rad} / \mathrm{sec})$ and for the yaw motion is about $\omega_{c y}=2 \cdot 10^{-2}(\mathrm{rad} / \mathrm{sec})$. These values are sufficient to reproduce acceleration/deceleration transitions and also steering maneuvres in normal driving situations.

\section{CONClusions AND Future WORKS}

\section{A. Conclusions}

Based on perceptual issues in driving a vehicle, we proposed a 2 DOF low cost platform for driving simulator which kept acceptable driving behavior and realism. Although these devices allowed partial restitution of dynamics inertial effects, pertinent cues have been taken into account, namely longitudinal and yaw motions also a steer wheel feedback not discussed in this paper.

The designed platform has two degrees of freedom. The first one makes it possible to drive the cabin of the simulator in a front/rear translation. The second makes possible to produce yaw rotations. The combination of the two DOF 
may give the illusion of acceleration variation and drive in curve.

In order to control in the best way, the dynamic model of the platform is developed. The parameters identification is not discussed in this paper. Therefore, a frequency characterization, given from the experimental study, allows us to give a principal conclusion that the platform is satisfactory from the velocity/acceleration limits and from the perceptual point of view. These findings are validated by real drivers completely satisfied with the motions fed back (longitudinal and yaw) quite acceptable.

\section{B. Future works}

In the perspective, we expand to identify completely the all platform parameters which can simply the choice of control strategy of the motions restitution. Also, we plan to make tests in closed loop way using the steer wheel force feedback system. These future works will enable us to conclude on the impact of yaw movement over simulator sickness.

\section{ACKNOWLEDGMENT}

The authors gratefully acknowledge the contribution of MSIS-INRETS and IEF-ORSAY teams.

\section{REFERENCES}

[1] Y. Miyamaru, G. Yamasaki, and K. Aoki, "Development of a motorcycle riding simulator," Society of Automotive Engineers of Japan, vol. 23, pp. 121-126, 2002.

[2] D. Ferrazzin, F. Barnagli, C. Avizzano, G. Pietro, and M. Bergamasco, "Designing new commercial motorcycles through a highly reconfigurable virtual reality-based simulator," Journal of Advanced Robotics, vol. 17, no. 4, pp. 293-318, 2003.

[3] L. Nehaoua, H. Arioui, H. Mohellebi, and S. Espié, "Motion Cueing Algorithms for Small Driving Simulator," in Proceedings 2006 IEEE International Conference On Robotics and Automation (ICRA06), Orlando, Florida, May 2006, pp. 3189-3194.

[4] P. Grant, B. Artz, J. Greenberg, and L. Cathey, "Motion Characteristics of the VirTtex Motion System," in Proceedings of the 1st HumanCentered Transportation Simulation Conference, IOWA, USA, 2001.

[5] L. D. Chen, Y. Papelis, G. Waston, and D. Solis, "NADS at the University of IowA: A tool for driving safety research," in Proceedings of the 1st Human-Centered Transportation Simulation Conference, IOWA, USA, 2001.

[6] G. Reymond and A. Kemeny, "Motion cueing in the RENAUlt driving simulator," in Vehicule System Dynamic, vol. 34, Paris, France, Oct. 2000, pp. 249-259.

[7] L. Sung, K. Ha, C. H. Cho, and L. J. Lee, "The Kookmin University Driving Simulators for Vehicle Control System Development and Human Factor Study," in Driving Simulation Conference (DSC04), Paris, France, 2004.

[8] G. Reymond, A. Heidet, M. Canry, and A. Kemeny, "Validation of RENAULT's Dynamic Simulator for Adaptive Cruise Control Experiments," in Proceedings of the Driving Simulator Conference (DSC00), Paris, France, 2000, pp. 181-191.

[9] M. Dagdelen, G. Reymond, A. Kemeny, and M. Bordier, "MPC based motion cueing algorithm: development and application to the Ultimate driving simulator," in Proceedings of the Driving Simulator Conference (DSC04), Paris, France, 2004.

[10] D. Stall and S. Bourne, "The National Advanced Driving Simulator: Potential applications to ITS and AHS research," in Proceeding of the 6th Annual meeting of the Intelligent Transportation Society, Washington D.C, USA, 1996, pp. 700-710.

[11] H. Mohellebi, S. Espié, H. Arioui, A. Amouri, and A. Kheddar, "Low cost motion platform for driving simulator," in 5th International Conference on Machine Automation (ICMA04), Osaka, Japan, Nov. 2004.
[12] L. Nehaoua, H. Mohellebi, A. Amouri, H. Arioui, and A. Kheddar, "Design and control of a small-clearance driving simulator," IEEE Transactions on Vehicular Technology, vol. 57, no. 2, pp. 736-746, 2008.

[13] L. Nehaoua, H. Arioui, H. Mohellebi, and S. Espié, "Restitution Movement for a Low Cost Driving Simulator," in Proceedings of the 2006 American Control Conference (ACC06), Minneapolis, Minnesota, June 2006, pp. 2599-2604.

[14] P. R. Grant and L. D. Reid, "Motion washout filter tuning: Rules and requirements," Journal Of Aircraft, vol. 34, pp. 145-151, Mar.-Apr. 1997.

[15] M. A. Nahon and L. D. Reid, "Simulator Motion-Drive Algorithms: A Designer's Perspective," Journal of Guidance and Dynamics, vol. 13, pp. 356-362, July 1989.

[16] M. Idan and M. A. Nahon, "Off-Line Comparison of Classical and Robust Flight Simulator Motion Control," Journal of Guidance and Dynamics, vol. 22, pp. 702-709, Sept.-Oct. 1999.

[17] L. Reid and M. Nahon, "Response of Airline Pilots to Variations in Flight Simulator Motion Algorithms," in Journal of Aircraft, vol. 25, July 1988, pp. 639-646.

[18] L. Nehaoua, A. Amouri, and H.Arioui, "Classic and Adaptive Washout Comparaison for a Low Cost Driving Simulator," in Proceedings of the 13th Mediterranean Conference on Control and Automation (MED05), Limassol, Cyprus, June 2005, pp. 586-591.

[19] J. Neimer, H. Mohellebi, S. Espié, and A. Kheddar, "Optimization of linear motion base dedicated to normal driving conditions," in Driving Simulator Conference (DSC05), Orlando, Florida, 30 nov-2 dec 2005.

[20] S. Marchant, "Réalisation d'un simulateur automobile," in Master Thesis, Ecole Nationale de l'Aviation Civile, France, Aug. 1995.

[21] R. Featherstone, Rigid Body Dynamics Algorithms. Springer, 2008. 